\title{
Observación y descripción. Una experiencia didáctica interdisciplinaria a partir del Proyecto Pedagógico de Aula
}

\author{
Observation and description. An interdisciplinary teaching \\ experience from the Classroom Pedagogical Project
}

\section{Melina Morillo}

morillomelina77@gmail.com

Instituto Montecarmelo, Venezuela

\begin{abstract}
- Artículo recibido en septiembre 2018 - Arbitrado en octubre 2018

RESUMEN

- Publicado en enero 2019

A través del Proyecto Pedagógico de Aula (PPA) se pueden realizar trabajos interdisciplinarios que proporcionen beneficios permanentes en los distintos niveles de la educación. En este caso, la investigación tuvo el propósito de incrementar en los estudiantes de 6to grado de Primaria los procesos de la observación y la capacidad para registrar mediante el texto descriptivo todas sus observaciones. Se desarrolló como un proyecto de acción con siete participantes, bajo un enfoque cualitativo. Se asentaron los procesos y resultados en registros anecdóticos y cuadernos de escritura espontánea. Asimismo, se utilizaron estrategias creativas y simuladores, para finalmente obtener el producto escrito de los estudiantes. Se concluye que las estrategias creativas dentro de un PPA, llevado a cabo por etapas, son altamente efectivas para dirigir a los estudiantes a observar, describir y experimentar en distintos escenarios pedagógicos.
\end{abstract}

Palabras clave: Proyecto Pedagógico de Aula; interdisciplinariedad, texto descriptivo, observación, simulación

\begin{abstract}
Through the Classroom Pedagogical Project (PPA), interdisciplinary work can be carried out that provides permanent benefits at different levels of education. In this case, the research was intended to increase the processes of observation and the ability to record all their observations through the descriptive text in 6th grade students. It was developed as an action project with seven participants, under a qualitative approach. The processes and results were based on anecdotal records and spontaneous writing books. Likewise, creative strategies and simulators were used to finally obtain the written product of the students. It is concluded that the creative strategies within a PPA, carried out in stages, are highly effective in directing students to observe, describe and experiment in different pedagogical settings.
\end{abstract}

Keywords: Classroom Pedagogical Project; interdisciplinarity, descriptive text, observation, simulation educative 


\section{INTRODUCCIÓN}

El aula de clase puede convertirse en un lugar de conquista si se plantea un proyecto con propósitos claros $\mathrm{y}$ procedimientos que incluyan la experiencia, las actividades lúdicas, las tareas, los contenidos curriculares y la interrelación de los temas a partir de un enfoque interdisciplinario.

$\mathrm{Al}$ combinar las disciplinas y lograr una interacción de los estudiantes con la materia, los objetivos y una visión prospectiva, el alcance puede llegar más allá del cumplimiento de una labor programática. Los jóvenes se ven implicados en una educación holística, bajo una guía que les reconoce dentro del pensamiento crítico, las posibilidades cognoscitivistas y humanistas y el logro del aprendizaje significativo en un ambiente de equidad.

El contexto en el que se realiza el proyecto es el Instituto Montecarmelo, de Caracas, un colegio en donde los jóvenes son educados en un proceso de aprendizaje basado en la experimentación, desde kínder. El colegio es una institución de matrícula reducida; la mayoría de las aulas cuentan con grupos de cinco a dieciocho estudiantes. Se trabaja con un sistema de aula abierta e integradora. Dentro de los rasgos de los estudiantes de la institución se encuentran niños sin discapacidades, jóvenes con síndrome del espectro autista, Asperger, síndrome de Down, entre otros. Se caracteriza por el desarrollo de novedosos métodos centrados en el respeto de las conductas infantiles, la incorporación de la expresión plástica, literaria y, en general, de la expresión creadora en la vida escolar. Su propia fundadora expresa que ha "buscado la educación por el juego, el arte, la literatura, la creatividad y la relación horizontal maestro-alumno" (Urdaneta, 2010). La idea de educación dentro del instituto es oponer la belleza que está en el interior de cada uno al entorno pedestre, a la pobreza cultural del ambiente, a la banalización y mercadeo del mundo infantil a causa de políticas erradas de muchos medios de comunicación y la explotación comercial, como rezan parte de sus principios y creencias.

Para tener éxito en las múltiples actividades deben contemplarse, en su enfoque pedagógico, las nociones del método Montessori (2004) que promueve un ambiente sencillo, limpio, artísticamente dispuesto para el desarrollo de los niños y niñas; asimismo, bajo este paradigma se promueve la solidaridad, el trabajo motivador, la responsabilidad, la libertad de expresión y las posibilidades de concentración en un lugar apto para su desempeño. En este tipo de responsabilidad, conviene recordar las enseñanzas de Feuerstein (Velarde, 2008) por sus orientaciones de creación de una 
inteligencia que pueda adaptarse a los cambios y que progresivamente, los estudiantes puedan asumir la flexibilidad y evolución que les permitan aportar beneficios a la sociedad; así también se recuerdan los principios de Delors (1996) para convertir la educación en un "aprender a conocer" o las investigaciones de Díaz Bajo (2019) que señalan las múltiples innovaciones que podrían combinarse. Del mismo modo, se han tomado en cuenta las nociones del eclecticismo ideológico y pedagógico (Rubio, 2019) del método de Carmen Lyra en donde se practica "un contínuum, una armonización de los pensamientos filosóficos de Federico Froebel, las hermanas Rosa y Carolina Agazzi, Ovide Decroly y por supuesto de María Montessori que buscan enaltecer y dar dignidad al infante como ser humano".

Dentro de las características de las aplicaciones didácticas que se llevan a cabo, deben destacarse las inclinaciones artísticas y la promoción permanente de las manifestaciones como la danza, las artes plásticas, la literatura, la expresión corporal, en general. En ese sentido, siempre se procura conectar el arte (desde la perspectiva del especialista que esté trabajando con los chicos) con el contenido pedagógico. En el caso de la investigadora se realizó la conexión con la literatura.
Con esta perspectiva se ha llevado a cabo dentro del Proyecto Pedagógico de Aula denominado "Los materiales y los fenómenos físicos" una intervención didáctica que tiene como propósito incrementar en los estudiantes de 6to grado de la escuela primaria los procesos de la observación y la capacidad para realizar el registro de lo observado.

El punto de partida para la práctica de la experimentación fue una actividad lúdica que comporta la descripción de uno de los compañeros, el registro personal en un cuaderno de redacción espontánea y la sistematización de la observación. Seguidamente, se replicó la actividad pero esta vez con el uso de simuladores para, a partir de allí, impulsar el aprendizaje de los contenidos relacionados con la materia, los estados de la materia, el átomo, la molécula. Posteriormente, se migró a los contenidos relacionados con los conceptos, componentes y conocimiento de las sustancias, mezclas, soluciones, concentraciones y finalmente a tratar de desentrañar los distintos fenómenos físicos, entre ellos la luz y el sonido.

La educación con simuladores es efectiva y segura (Biencinto, Domínguez y García, 2005; Kramer, 2002) y ayuda a los docentes a establecer vínculos entre la teoría y la práctica y sobre todo apoya a los maestros a planificar la evaluación 
(Rodríguez-Miñambres et al. 2018). Con el uso de los simuladores se aplica un trabajo de representaciones mediante algoritmos, software y modelos que simplifican las relaciones de sistemas (Vega, 2012).

Con estos lineamientos, se han aplicado dos experiencias con simuladores adaptadas de las propuestas por University of Colorado Boulder (2019): una sobre la estructura del átomo y otra sobre los estados de la materia. Luego, se han llevado las actividades a otro nivel de práctica en el aula. Como alegan Garófalo, Chemes y Alonso (2016), si bien las simulaciones "no son un sustituto de la observación y la experimentación de fenómenos reales, constituyen un modelo para facilitar la interpretación de tales fenómenos”. De esta forma, se han convertido en un medio valioso para explorar distintas facetas de los fenómenos experimentales y actividades que ayudan a comprender procesos de la ciencia y la cultura con mayor amplitud.

Efectivamente, Reyes, Mayorga y Bustos (2017) afirman que es necesario "contribuir a la desmitificación del pensamiento infantil como un pensamiento ajeno a las representaciones y a las construcciones mentales con sentido" (p. 967). Los autores, a partir de un enfoque por analogías privilegian el proceso de observación combinado con la reflexión de todas las cosas que rodean a los niños, de tal forma que se logre un acercamiento a los fenómenos naturales para establecer semejanzas, realizar predicciones y conjeturas además de entablar un diálogo entre lo vivido $y$ el surgimiento de las emociones. Estas analogías asisten a los niños para reconocer el mundo y apoyan en el desarrollo de competencias para interpretar textos.

Se aspira que los estudiantes desarrollen mucho más que habilidades de observación y que manifiesten la disposición a integrar lo aprendido en diversas áreas con sus experiencias cotidianas, hasta llegar a apropiarse de valores y saberes que puedan llevar a su vida diaria $y$ transferirlas a sus necesidades personales y colectivas. Asimismo, se aguarda que contribuyan a desarrollar un pensamiento crítico $y$ creativo que les impulse a pensar por sí mismo y puedan afrontar los cambios en su promoción a años superiores, mediante la elaboración de textos descriptivos y la consignación en registros personales.

Se decidió especialmente no trabajar las teorías sobre descripción de forma previa al experimento, con un esquema de trabajo de enseñanza de la lengua con prácticas precedentes y elementos $\mathrm{y}$ conceptos postreros, para lograr el aprendizaje. Dentro del programa de gramática, se trabajan las distintas categorías y se conectan con los tipos 
textuales. Si la categoría estudiada es el verbo, por ejemplo, se estudia el texto narrativo. En este caso se privilegió la descripción para acercarse a los procesos de observación científica.

\section{El Diccionario de la Lengua Española,} DLE (https://dle.rae.es/) dice sobre el verbo describir en su primera acepción: Representar o detallar el aspecto de alguien o algo por medio del lenguaje y en su tercera acepción puntualiza que la descripción es: Definir imperfectamente algo, no por sus predicados esenciales, sino dando una idea general de sus partes o propiedades. Precisamente, se utiliza el texto descriptivo porque se conecta con la categoría de los adjetivos y a partir de estos se enlaza con los distintos recursos literarios. Igualmente, al abordar la descripción es necesario el trabajo con la sinonimia y la antonimia y llegar a entender que algunas palabras no poseen sinónimos absolutos. Es el caso de los términos "blanco" y "negro"; solo cuentan con palabras que tienen un significado proximal y partiendo del concepto de que existen términos que pueden expresar un significado similar, se construyen algunos recursos literarios. Esto permitió tomar elementos de la literatura y la adjetivación para abordar la creación de descripciones y que los estudiantes produjeran el texto sin conocer anticipadamente la superestructura textual del texto descriptivo. Gracias a ello, la conceptualización y práctica de este tipo de texto, se convirtió en un proceso donde los estudiantes fueron los protagonistas.

Ciertamente, realizar informes propios siempre favorece la reflexión destrabada de las prácticas tradicionales y de los estereotipos (Bombini, 2018) y permite desplegar los intereses personales para luego vincularlos con los trabajos relacionados con las prácticas curriculares. Es así como se logrará, en los alumnos que están próximos a graduarse de su escuela Primaria, el propósito de desarrollar las competencias de observación y de reportar lo observado mediante el texto descriptivo.

\section{MÉTODO}

La metodología respondió a un enfoque cualitativo, bajo un paradigma interpretativo (Hernández, Fernández y Baptista, 2010) dentro de una investigación de campo, con la modalidad específica de Informe de Proyecto de Acción (UPEL, 2016, p. 18) que contempla aproximaciones etnográficas, debido a la participación activa de la investigadora. El tipo de trabajo se realizó sobre la práctica con una propuesta llevada a cabo desde el Proyecto de Aula.

La recolección de información se operó mediante la estrategia de la observación participante por cuanto se acopiaron los 
registros "de las acciones, los discursos y la vida cotidiana de un grupo social" (Yuni, Urbano y Ciucci, 2005, p. 185).

Los testimonios de los estudiantes se recogieron en el mismo momento de la actividad en un proceso en continuo, como se procede en la investigación cualitativa. Así lo afirman Taylor y Bogdan (2002):

A lo largo de la observación participante, la entrevista en profundidad y las otras investigaciones cualitativas, los investigadores siguen la pista de los temas emergentes, leen sus notas de campo o transcripciones y desarrollan conceptos y proposiciones para empezar a dar sentido a sus datos.

En este trabajo se buscó darle sentido a toda la información recogida en el sitio y a los textos producidos por los estudiantes en su experiencia didáctica. Ello precisó de organizar la información y buscar "aquellas unidades de análisis que nos parecen relevantes (...) descubrir lo verdaderamente importante: el significado que se esconde tras los datos y dar sentido a los fenómenos (...) el investigador debe mantenerse firme y orientado al objeto de estudio" (Rodríguez y Valldeoriola, 2009, p. 72).

Los instrumentos utilizados para la recolección de información fueron un cuaderno de observación y un registro anecdótico validados por dos expertas en metodología y especialistas en Lengua y Literatura, además del cuaderno personal de redacción espontánea de cada uno de los estudiantes, que se trata como elemento íntimo y de uso privado.

La validez de constructo y de contenido respondió a los objetivos planteados, a la redacción y a la organización de las etapas de recolección de información. En el Cuadro 1 se muestra la rejilla de registro de la docente:

Cuadro 1. Elementos del registro anecdótico por cada uno de los estudiantes

\begin{tabular}{|c|c|c|c|}
\hline \multirow[t]{4}{*}{ Estudiante } & Actividad & \multirow{2}{*}{$\begin{array}{l}\text { Dimensión } \\
\text { Cognoscitiva }\end{array}$} & Expresión \\
\hline & Observación y descripción & & Actividad grupal \\
\hline & de mi compañero o & Actitudinal & Atención focalizada \\
\hline & compañera & Procesual & $\begin{array}{l}\text { Escritura } \\
\text { (descripción) }\end{array}$ \\
\hline & Simulación 1: Estructura del & Cognoscitiva & La observación \\
\hline & átomo (observación y & Actitudinal & Atención a los elementos \\
\hline & descripción) & Procesual & Descripción orgánica \\
\hline & Simulación 2: Estados de la & Cognoscitiva & La observación \\
\hline & materia (observación y & Actitudinal & Atención a los elementos \\
\hline & descripción) & Procesual & Descripción orgánica \\
\hline
\end{tabular}


La actividad se llevó a cabo por etapas, como corresponde con el tipo de estrategia de elaboración de un programa para el Proyecto Pedagógico de Aula (PPA), con enfoque interdisciplinario, en todas sus partes (adaptado de Arteaga Quintero, 2005):

- Nombre del PPA: "Los materiales y los fenómenos físicos"

- Docente: Melina Morillo

- Temas: la materia, sus propiedades y estados; la observación y las capacidades descriptivas

- Propósito: incrementar en los estudiantes de 6to grado de la escuela Primaria los procesos de la observación y las competencias para registrar en un texto descriptivo todo aquello que se ha podido observar

- Objetivos: (1) internalizar el proceso de observación con mayor sistematicidad; (2) desarrollar habilidades para registrar los datos obtenidos producto del proceso de observación; (3) expresar de una forma ordenada y coherente todos los resultados de un proceso de observación para realizar en el futuro la experimentación.

- Contenidos y áreas: Ciencias Naturales: la observación y la experimentación. Lengua y Literatura: el texto descriptivo, las figuras literarias, el símil.
- Participantes: Un grupo de sexto grado que se encuentra próximo a ingresar al bachillerato. Está conformado por siete niños: cinco niñas y dos niños, todos de once años de edad. En el grupo intervenido en este proyecto, una de las niñas sufrió una parálisis que disminuyó algunas de sus capacidades, sin embargo se ha superado satisfactoriamente.

- Ambiente: La actividad se llevó a cabo en el salón de computación, debido a que es un grupo pequeño y este espacio disponible, ya conocido por los estudiantes, fue suficiente y acorde para el adelanto de la actividad.

- Duración: el proyecto se cumplió por etapas durante todo el segundo lapso de clases, aproximadamente dos meses. Su extensión se debió al encadenamiento de distintas actividades e integración de disciplinas que ampliaron las fases interdisciplinarias del proyecto: arte, ciencias naturales, ciencias sociales, lengua y literatura (Arteaga Quintero, 2019).

- Evaluación: antes, durante y después (proceso y producto). En este aparte, debe resaltarse que para no olvidar el registro de los testimonios que constituye el proceso de cierre del experimento, se ofreció a los estudiantes una herramienta que les resultara práctica y estimulante para 
generar el producto final, en el que reflejen el resultado de su experiencia. Por eso, se consideró que el texto descriptivo podía funcionar; porque, no representaba un esquema restrictivo para el estudiante. Motivado a esto fue necesario evaluar la efectividad de este tipo de textos en el protocolo del proceso de observación, en las actividades previas, y de esta forma determinar si la creación de este texto puede favorecer en la fase práctica del proyecto de aula.

A partir de las experiencias previas se recolectaron siete textos, de los cuales se presentan dos muestras en los resultados. Luego se presentan dos textos de la tarea final con simuladores.

- Actividad central como proceso preliminar para realizar una observación previa a la experimentación: "Observación y descripción de mi compañero o compañera”

i. Se formularon a los estudiantes una serie de preguntas orientadas a qué hacen o cómo discriminan para observar en un proceso planificado; asimismo, se les instruyó sobre lo que debían observar en una situación de experimentación. Se les indicó que deben estar muy vigilantes por cuanto a que durante los experimentos van a tener que enfocar su examen hacia ciertos procesos y prestar atención a los cambios que a veces parecen imperceptibles pero que, sin duda, pueden suceder y que deben apreciar $\mathrm{y}$ posteriormente registrar de manera escrita para poder internalizar lo que sucedió en la experiencia con el fin de aprender el contenido previsto en la planificación curricular.

ii. En el procedimiento se hizo acopio de estrategias de acercamiento al grupo (integración horizontal en donde el docente se coloca a la par de los estudiantes; demostración de la misma curiosidad por entender el proceso; narración de haber participado antes en experiencias similares y la gratificación que genera, etc.) para lograr que los estudiantes centren su atención en un objeto o proceso determinado. Otra de las preguntas que se formuló la propia docente está vinculada con la actitud de los estudiantes debido a que no existe forma de saber, exactamente, qué están mirando cuando están mirando, o cómo conocer la perspectiva con la que los niños captan lo que ven. Esta actividad proporcionó elementos que permiten tener una orientación acerca de cómo enfocar el proceso de experimentación. Otro de los cuestionamientos era el diseño de un instrumento para registrar la información de la experiencia, debido a que en oportunidades anteriores se había trabajado con un formato que 
los estudiantes no consideraban del todo valioso.

iii. Las instrucciones generales fueron respeto mutuo, silencio y concentración.

iv. La experiencia previa:

Debido a que el grupo tiene un número impar de integrantes (siete), fue preciso contar con la presencia de una invitada (la secretaria de la coordinación quien también es docente) para que sirviera de objeto de observación y observara a alguno de los estudiantes. La instrucción inicial fue que se despojaran de cualquier objeto -solamente si este les pudiera causar molestias-, como suéter, reloj, collares, pulseras $\mathrm{y}$ debían dejarlas fuera del salón. La siguiente instrucción fue que se descalzaran, porque, parte de la actividad era observar la mayor cantidad de los rasgos de la otra persona. Una de las niñas no quiso quitarse las medias, aunque sí se quitó los zapatos.

Al encontrarse en el salón se les pidió que se sentaran en el suelo en forma circular. Se implementó un ejercicio de relajación, aunque no en el sentido estricto de este tipo de ejercicios. Pero, sí llevaba la intencionalidad de conectar con determinadas emociones, en especial las sensaciones de tranquilidad, concentración y disciplina armónica. Se les pidió que cerraran los ojos e hicieran un par de respiraciones. Posteriormente, se les pidió que recordaran el momento de llegada al colegio en la mañana, a qué compañeros vieron primero, quiénes se encontraban allí al llegar (todo esto con los ojos cerrados). Se continuó interrogándoles sobre una vez que llegaron a su salón dónde se sentaron; si se sentaron en el mismo lugar donde se sientan habitualmente; quién estaba sentado a su derecha o a su izquierda. Luego, debían enfocarse en el compañero que estaba a su derecha en el preciso momento de la actividad. Si recordaban cómo estaba peinado, cómo estaba vestido, si llevaba algún elemento peculiar que no suele traer a diario, si notaron si el compañero tiene alguna marca particular como un lunar, una peca en la mejilla derecha, o en la mejilla izquierda.

Una vez que se les dio ciertas pistas para saber si ellos habían observado con detenimiento, se les pidió que abrieran los ojos y tomaran de la mano al compañero a su derecha. Se les invitó a organizarse en parejas para observar detenidamente a su compañero. Todo esto bajo la norma de que este es un ejercicio de silencio, mientras la maestra no diera otra instrucción, en el momento cuando, como parte del ejercicio, los estudiantes debían decir ciertas frases para que sus compañeros pudieran "observar" su voz. 
El proceso de observación implicaba ir fijando la atención localizada en el compañero. Primero, observamos el cabello, lo tocamos con todo respeto; observamos la frente, para precisar si con algún gesto se formaban líneas. Se les pidió que representaran una cara de molestos, que se sonrieran, que le hiciera una mueca al compañero y este a su vez debía fijar la atención en la parte del rostro que estábamos observando. Previamente se dio la instrucción de que se denominaría a cada miembro de la pareja como A o $\mathrm{B}$ y mientras A gestualizaba, B tenía que observar. Después se intercambiaban los roles. En medio del ejercicio se les pidió decir unas frases o entonar el fragmento de una canción al oído del compañero y que el compañero escuchara el sonido de su voz.

Posterior a la observación y para no perder la conexión con lo observado, se les pidió continuar en silencio absoluto, sin emitir ningún tipo de sonido, para dedicarse a producir un texto. Colocamos música de fondo muy suave para permitirles escribir. En esta instancia, la docente volvió a formularse a sí misma la interrogante: ¿Cómo lograr que el proceso de registro de la observación sea lo más orgánico posible?

Para lograrlo se sugirió a los estudiantes que no solo relataran lo que hicieron debido a que esto generaría la confección de un discurso cuya superestructura textual sería la propia del texto narrativo, y como tal la más natural en la expresión cotidiana. Precisamente, se les pidió que expresaran las características de la persona que se encontraba frente a ellos, sin explicarles previamente las teorías del texto descriptivo. Así se podría apreciar en qué fijaron su atención en los determinados momentos de la experiencia, reproduciendo de alguna forma toda su observación. La revisión de los párrafos descriptivos ya elaborados llevó a la explicación y comentarios sobre la adjetivación, las figuras literarias, el símil, la metáfora y la inclusión de las emociones en el discurso, así como otros temas propios de la Lengua y la Literatura. Luego de procesar las creaciones en sus cuadernos de escritura espontánea se comenzó con la siguiente práctica.

Al trabajar con el simulador, se trató de emular la actividad realizada en la observación entre los compañeros; tanto en el proceso de silencio en los momentos de concentración como de compartir las apreciaciones; de esta forma los estudiantes lograron registrar los resultados de su interacción con el simulador.

- Actividades siguientes por simuladores: a y b

(a) Reconocimiento de la estructura del átomo (ver Gráfico 1): se fueron 
construyendo los átomos de distintos elementos de la tabla periódica en el simulador. A través de esta experiencia, los estudiantes pudieron apreciar cuando el átomo está ionizado, cuando tiene carga neutra, donde se ubican los electrones, los neutrones y los protones. Se les pidió que observaran con cuidado cada una de sus partes y sus cambios en la medida de que se iban realizando las modificaciones. Al final debían describir los objetos presentes y la propia experiencia.

(b) Los diferentes estados de la materia (ver Gráfico 2): se realizaron las actividades tal como se ofrecen mediante el simulador. Luego de observar atentamente se les pidió describir cada estado de la materia; los componentes, la forma de realizar la actividad; la actitud de los compañeros y la experiencia propia.

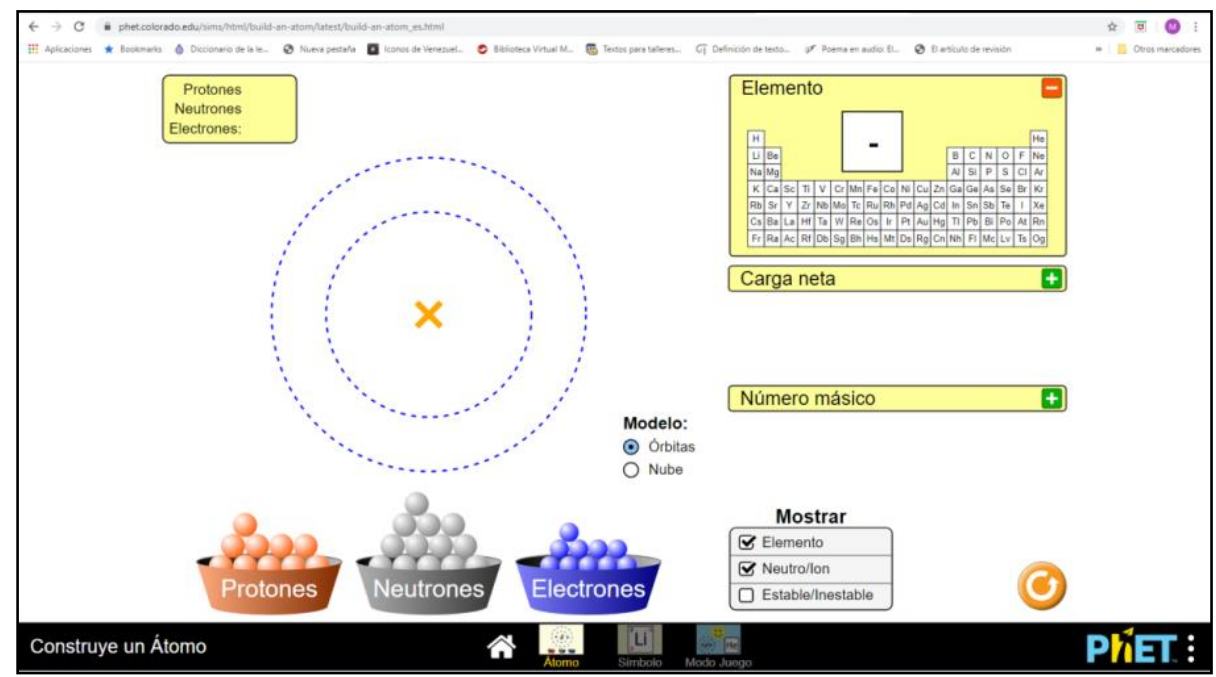

Gráfico 1. Construye un átomo. Actividad (a). (Fuente: Phet.Colorado.Edu)

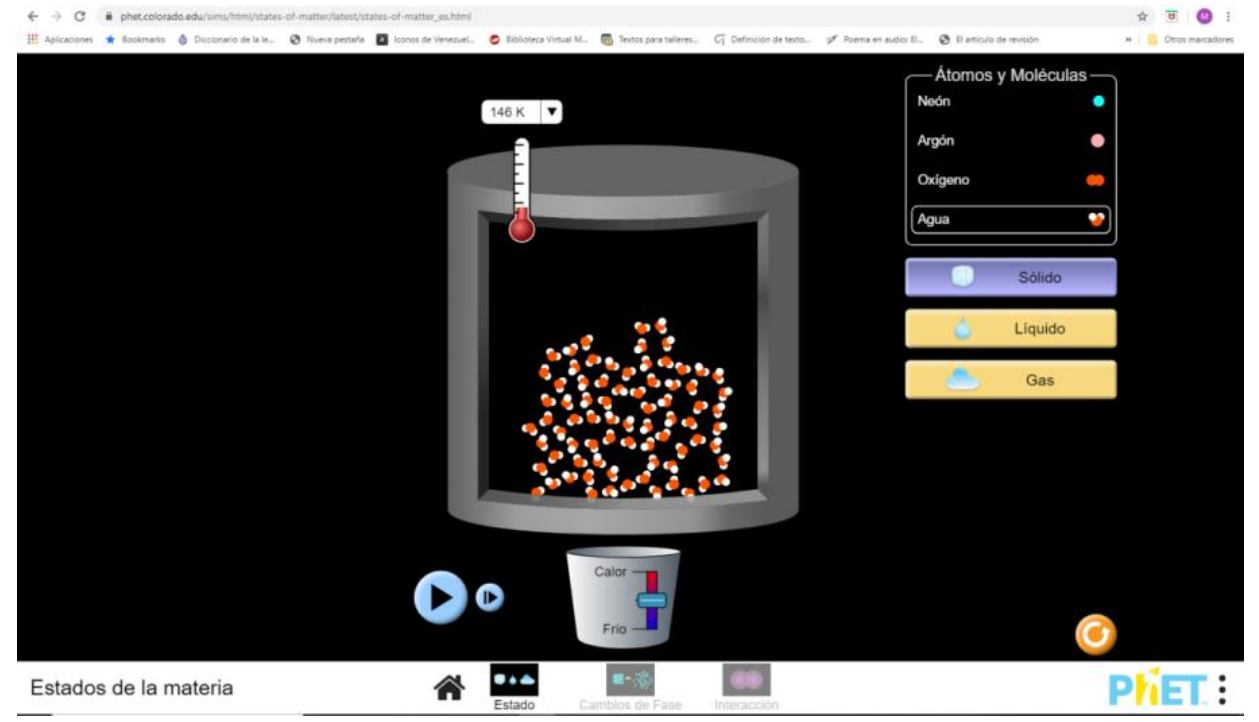

Gráfico 2. Estados de la materia. Actividad (b). (Fuente: Phet.Colorado.Edu) 
- Divulgación: El último paso del Proyecto Pedagógico de Aula es divulgar los logros, para ello se ha planificado: elaboración de una cartelera de aula; preparación de diversos blogs en donde se publiquen en la WEB los textos producidos por los estudiantes y una presentación del docente, mediante una charla, así como una publicación de artículo de investigación.

\section{RESULTADOS}

Las derivaciones arrojaron materiales interesantes para este grupo que está encaminándose al bachillerato ya que las asignaturas que cursarán en esa etapa demandarán competencias en experimentación. También deben tener habilidades para registrar la información que se vivencia. La estrategia de observación del compañero produjo frutos sugestivos que luego pudieron ser muy útiles en los experimentos con simuladores.

En su cuaderno de redacción, los estudiantes compusieron los textos en el marco de una sección de la planificación denominada Escritura Espontánea. Regularmente, se les proporciona un "reactivo" o consigna y los muchachos generan textos con distintas estructuras: narración, versos, descripción, conectadas con la visión del proyecto. En esta "escritura guiada" se les proporciona un tiempo limitado para formar figuras literarias e introducirlas dentro de un texto, generando un esquema bien interesante a la hora de escribir de forma sistemática pero imaginativa. Esto les proporciona pericia a la hora de crear textos con libertad.

En este caso, para trabajar con los elementos de la escritura creativa, se les indicó que debían describir por escrito a sus compañeros (ver Cuadro 2 y Cuadro 3).

\section{Cuadro 2. Descripción realizada por Carmina (11 años)}

\section{Texto descriptivo 1}

Zoe tiene el cabello castaño oscuro; tan largo, tan largo que siento el cabello largo. Sus ojos son especialmente alegres de un color marrón como chocolate, tienen forma almendrada y me veían con curiosidad. Sus pestañas son muy largas y sus cejas son muy gruesas.

Su nariz no es muy pequeña ni muy grande, no es ancha ni fina, no es larga ni corta, es simplemente su nariz. Su boca es de labios ni muy gruesos ni muy finos. Su sonrisa es cálida y solo con sonreírte, hace que tú sonrías también. Cuando la miro, siento la alegría que transmiten sus ojos. Tiene una sonrisa contagiosa y cada vez que la veo sonrío.

Su piel es como un coral debajo del agua. Lleva el uniforme del colegio. Sus medias son azules y sus pies pequeñitos y sus dedos estaban arrugados. Sus manos no tenían ninguna marca en especial. Pero, son lindas y parecen muy suaves.

Fuente: Elaboración propia a partir de la escritura de un estudiante 


\section{Cuadro 3. Descripción realizada por Jaime (11 años)}

Texto descriptivo 2

Manu, tiene el pelo negro y corto. Tan corto como una leona. Sus ojos de color café (muy lindos), son oscuros como la noche y me recuerdan cosas hermosas. Sus pestañas eran largas, muy largas se mueven sin parar. La nariz la tiene pequeñita pero ancha y la hace lucir muy linda.

Manuela tiene una boca pequeña y de un color rosa oscuro. Su sonrisa es grande. Al mirar su barbilla, me doy cuenta de que es más alargada que redonda. Casi no arruga la frente, porque siempre está contenta.

$\mathrm{Al}$ mirar sus manos, me di cuenta de que son oscuritas como el cacao, tienen rayas como la de la vida muy larga y me recuerdan a las de Minie Mouse. Sus uñas son cortas y sus dedos son finitos y sus uñas son muy cortas.

Su piel es como la de mi mamá. Lleva puestos unos zarcillos uno es de estrellas y el otro es de flor. Lleva un suéter gris muy grande que en verdad le queda bien. Cuando la miro, recuerdo a mis amigos. Cada vez que la oigo, recuerdo a mi maestra Grisel. Sus zapatos son geniales y ella es mi amiga.

Fuente: elaboración propia a partir de la escritura de una estudiante

Seguidamente, al culminar las actividades de simulación se realizaron las descripciones pertinentes con el mismo entusiasmo de los textos preliminares (ver Cuadro 4 y Cuadro 5)

\section{Guadro 4. Descripción de "Reconocimiento de la estructura del átomo"}

\section{Texto descriptivo 3}

Lo primero que observé fue la estructura del átomo. Tiene un núcleo y la corteza. Al combinar sus elementos (protones, neutrones y electrones) aparece el nombre. Puede verse su estructura en forma de nube o con las órbitas de los electrones que este tiene. Al observar la carga del átomo, descubrimos que esta solo varía dependiendo de sus electrones y protones; los neutrones no afectan su carga. En la balanza se ve la cantidad de elementos presentes en el núcleo. Cada elemento de la tabla periódica posee un número distinto de electrones, protones y neutrones. El último átomo que construimos fue el neón y tenía ocho protones, electrones y neutrones. 


\section{Cuadro 5. Descripción de "Los diferentes estados de la materia”}

Texto descriptivo 4

Observamos moléculas de distintos elementos (neón, argón, oxígeno y agua). Sólo la molécula de agua tenía tres átomos dos de hidrógeno y uno de oxígeno los otros elementos tenían un solo átomo representado. Cada elemento tenía un color distinto para representarlo. Cuando los materiales estaban en estado líquido, las moléculas se movían suavemente, se desplazaban por todo el recipiente. Cuando se encontraban en estado sólido, su movimiento era escaso, casi no se notaba el movimiento; sobre todo en el caso del agua; parecían tener pequeños temblores y ocupaban el mismo lugar; los gases se aprecian más compactos que el agua. Pero, al observar su comportamiento, mientras estaban en estado gaseoso, se agitaban mucho y se movían por todo el recipiente, incluso disminuían su número. La temperatura era variable.

Como ha podido constatarse, los estudiantes fueron capaces de describir el proceso de trabajo sobre la materia y sus propiedades y se apoyaron en la primera experiencia realizada.

Con la guía del proceso de observación en el uso de los simuladores $y$ al proporcionarles un esquema de apreciación ordenado, fijaron su atención en distintos aspectos que les permitieron comprender el papel de cada uno de los elementos de la estructura del átomo en la configuración de la materia. En el caso de los estados de la materia, pudieron enfocar su atención hacia el comportamiento de los materiales de acuerdo con los estados y la introducción de circunstancias que podían variar las condiciones iniciales. Lo más importante fue que al apreciar ciertos detalles pudieron inferir información. Por ejemplo, establecieron la conclusión de que si todo es materia, todo tiene carga eléctrica; que a menor temperatura, los movimientos son menores para preservar energía; cuando la temperatura aumenta, estamos desprendiéndonos de energía como le sucedió al agua al evaporarse.

En el Aprendizaje Basado en Proyectos (ABP) tal como lo apunta CaeiroRodríguez (2018) la actividad a realizar es una aventura "tanto para el maestro, el profesor, el niño o la propia institución educativa que se transforma incluso medioambientalmente" (p.164). Es una forma de afrontar la enseñanza de manera dinámica y con la certeza de que no hay una serie de temas sucesivos sin ninguna conexión. En un proyecto de aula, existen múltiples posibilidades, desde la intervención de Kilpatrick (1925) hasta la estructuración de Triana Castro en "profundización, recreación y reflexión” (2018, p. 231) que propician las relaciones de las actividades de aula con la vida de los jóvenes y con las posibilidades de no entender la enseñanza como actividades descontextualizadas de su mundo. De 
esta forma, el grupo intervenido pudo realizar un proceso organizado y fijar aprendizajes conectados con su realidad, además de sentir que era su propio descubrimiento mediante la observación y descripción.

Igualmente, la actividad llevada a cabo por los estudiantes del Instituto Montecarmelo propició una forma de ver la enseñanza con autonomía. Sobre ello Johari y Bradshaw (2008) afirman que en este tipo de aprendizaje "el alumnado toma las riendas de su aprendizaje" por lo que los profesores deben estar atentos para comprometerse con su grupo y los resultados para que todos "encuentren el equilibrio entre la habilidad y el desafío, desencadenando una experiencia agradable en el aprendizaje".

Es fundamental, certificar que las estrategias, objetivos y contenidos se encuentran previstos en el currículo y no son actividades periféricas 0 extracátedra. Según Thomas (como se citó en Sánchez, 2016) los proyectos deben ser la médula curricular, no un tema adyacente de tal forma que los estudiantes logren aprender los contenidos esenciales de una forma distinta a lo que se ha planificado en clases tradicionales. En las actividades realizadas como parte del Proyecto de Aula a cada estudiante le fue indicado que los contenidos aprendidos y la experiencia internalizada cumplía con la parte central del currículo de su año escolar, lo que les produjo gran satisfacción.

Finalmente, la experiencia con el texto descriptivo como parte de una estrategia para registrar la observación y comenzar la experimentación debe contar con el fundamento epistemológico de que la descripción no garantiza la objetividad ni tampoco se puede negar, desde la apertura, una subjetividad interpretativa para todo fenómeno observado. Así, lo afirman Aguirre y Jaramillo (2015) al señalar que "el éxito de la descripción estaría en que lo descrito correspondiera de modo fiel (...) con el objeto, lo que presupone que el objeto es transparente a quien describe y a quien se describe" (p.177). Pero en las experiencias descritas, juega un papel muy importante la subjetividad y la libertad de la descripción, precisamente, en la búsqueda de una visión personal, para a la postre aprender a diferenciarla de las descripciones de hechos concretos y sus propiedades físicas.

\section{CONCLUSIONES}

Luego de haber trabajado con un grupo de estudiantes de 11 años, en el Instituto Montecarmelo con la aplicación de un Proyecto Pedagógico de Aula se logró incrementar en los estudiantes de 6to grado las técnicas, métodos y procesos de la observación $y$, seguidamente, se llegó a incrementar las competencias 
para escribir ordenadamente un texto que funcionara como el registro de toda la práctica.

Las posibilidades de observar a partir de una actividad personal, íntima, interesante y espontánea produjo en los estudiantes la disposición para replicarla en tareas con temas de la clase estipulados en el programa. La experiencia previa estimuló en el grupo el deseo de aplicar su vivencia a contenidos diferentes con lo que se logró una mejor observación y una descripción orgánica que les llevó a producir un texto descriptivo valioso para la experimentación.

El Proyecto Pedagógico de Aula fue una estrategia acertada para llevar adelante una tarea en distintas facetas, con la relación de disciplinas, específicamente elementos de las ciencias naturales y la literatura, además de brindarles a los estudiantes competencias de observación, experimentación y de producción escrita que les serán de utilidad al pasar al próximo nivel educativo.

\section{REFERENCIAS}

Aguirre, J.C. y Jaramillo, J.G. (2015). El papel de la descripción en la investigación cualitativa. Cinta moebio, 53, 175-189. Recuperado de https://scielo.conicyt.cl/pdf/cmoebio/ n53/ar06.pdf
Arteaga Quintero, M. (2005). La pedagogía humanista, el enfoque transversal y el uso del texto escolar. En Arteaga Quintero, M., Ayala, M. y García, C. SEMINARIO: El enfoque transversal en el diseño curricular, la pedagogía humanista y el uso del texto escolar, (5-25). Caracas: Editorial Salesiana

Arteaga Quintero, M. (2019). El mundo animal en la Gran Caracas. Práctica pedagógica transdisciplinaria a través del texto argumentativo. Caracas, Venezuela: Universidad Pedagógica Experimental Libertador. Recuperado de https://www.academia.edu/40756126/ El_mundo_animal_en_la_Gran_Carac as._Pr\%C3\%A1ctica_pedag\%C3\%B3g ica_transdisciplinaria_a_trav\%C3\%A9 s_del_texto_argumentativo

Biencinto, C., Domínguez, G. y García, J. A. (2005). La necesaria imbricación entre aprendizaje y formación. El simulador situativo como tecnología adecuada para la formación de formadores. Revista Complutense de Educación, 16(2), 645-671. Recuperado de https://revistas.ucm. es/index.php/RCED/article/viewFile/R CED0505220645A/16001

Bombini, G. (2018). Didáctica de la lengua y la literatura: entre la intervención y la investigación. Bellaterra Journal of Teaching \& Learning Language \& Literature, 11(4), 5-20. Recuperado de https://doi.org/10.5565/rev/jtl3.784

Caeiro-Rodríguez, M. (2018). Aprendizaje Basado en la Creación y Educación Artística: proyectos de aula entre la metacognición y la metaemoción. Arte, Individuo y Sociedad, 30(1), 159-177. Recuperado de http://dx.doi.org/10.5209/ARIS.57043 
Delors, J. (1996). La Educación encierra un Tesoro. París: UNESCO. Recuperado de http://www.unesco. org/education/pdfDELORS_S.PDF

Díaz Bajo, M. (2019). Panorama actual de las pedagogías alternativas en España. Papeles Salmantinos de Educación, 23. Recuperado de https://summa.upsa.es/viewer.vm?id= 108394

Garófalo, S., Chemes, L. y Alonso, M. (2016). Propuesta didáctica de enseñanza con simulaciones para estudiantes del profesorado en Ciencias Biológicas. Revista Eureka sobre Enseñanza y Divulgación de las Ciencias, 13(2). Recuperado de https://www.redalyc.org/jatsRepo/920/ 92044744009/html/index.html

Hernández, R., Fernández, C. y Baptista, P. (2010). Metodología de la investigación $5^{\mathrm{a}}$ ed. México: McGraw-Hill

Johari, A. y Bradshaw, A. C. (2008). Project-based learning in an intership program: A qualitative study of related roles and their motivational attributes. Educational Technology Research and Development, 56, 329-359

Kilpatrick, W. (1925). Foundations of method. Informals talks on teaching. New York, EUA: Macmillan. Recuperado de https://babel. hathitrust.org/cgi/pt?id=mdp.390150 57278676;view=1up;seq=7

Kramer, F. (2002). Manual práctico de educación ambiental: técnicas de simulación, juegos y otros métodos educativos. Madrid: Los Libros de la Catarata

Montessori, M. (2004). La mente absorbente del niño. México: Editorial Diana
Reyes, J., Mayorga, C. y Bustos, E. (2017). Construcciones analógicas sobre la lluvia en la primera infancia. $X$ Congreso Internacional sobre Investigación en Didáctica de las Ciencias, Sevilla, España. Recuperado de https://ddd.uab.cat/pub/edlc/edlc _a2017nEXTRA/55_-

_Construcciones_analogicas_sobre_la lluvia_en_la_primera_infancia.pdf

Rodríguez, D. y Valldeoriola, J. (2009). Metodología de la investigación. Cataluña, España: Universitat Oberta de Catalunya

Rodríguez-Miñambres, P., Rico-Martínez, A., López de Sosoaga, A. y Ugalde Gorostiza, A. I. (2018). Cómo evaluar una simulación de la metodología por proyectos a través del uso de rúbricas en el Grado de Educación Primaria. Revista Electrónica Interuniversitaria de Formación del Profesorado, 21(2), 43-63. Recuperado de http://www.aufop.com /aufop/revistas/lista/digital

Rubio, C. (2019). Carmen Lyra, maestra: tras las huellas de un eclecticismo pedagógico. Revista Educación, 43(2), Recuperado de http://www. redalyc.org/articulo.oa?id $=440581580$ 34

Sánchez, J. (2016). Qué dicen los estudios sobre el Aprendizaje Basado en Proyectos. En actualidad pedagógica.com. Recuperado de http://www.estuaria.es/wpcontent/uploads/2016/04/estudios_apr endizaje_basado_en_proyectos1.pdf

Taylor, S. y Bogdan, R. (2002). Introducción a los métodos cualitativos de investigación. México: Paidós

Triana Castro, Y. M. (2018). De la asignatura al taller de literatura 
como estrategia didáctica. Una reflexión desde la práctica docente. Enunciación, 23(2), 225-236. Recuperado de http://doi.org/ 10.14483/22486798.12732

University of Colorado Boulder. (2019). Phet Interactive Simulations. Recuperado de https://phet.colorado. edu/es/simulation/build-an-atom

Urdaneta, J. (2010). Instituto Montecarmelo. Recuperado de http://www.institutomontecarmelo.co $\mathrm{m} / \mathrm{q}$ enessomos.htm

Vega, J. (2012). La simulación en el aula. Contextos imaginarios en la enseñanza de ELE. (Trabajo de maestría), Universidad de Oviedo, España. Recuperado de http://digibuo.uniovi.es/dspace/bitstre am/10651/4016/6/TFM_Joaqu\%C3\%A DnVegaGonz\%C3\%A1lez.pdf

Velarde, C. (2008). La teoría de la modificabilidad estructural cognitiva de Reuven Feuerstein. Investigación Educativa, 12(22), 203 - 221

Yuni, J., Urbano, C. y Ciucci, M. (2005). Mapas y herramientas para conocer la escuela. Investigación etnográfica e Investigación-acción. Córdoba, Argentina: Editorial Brujas 